01

\title{
Ab initio моделирование энергии растворения и активности углерода в ГЦК-Fe
}

\author{
() Я.М. Ридный, А.А. Мирзоев, Д.А. Мирзаев
}

Южно-Уральский государственный университет (Национальный исследовательский университет), Челябинск, Россия

E-mail: ridnyiim@susu.ru

(Поступила в Редакцию 5 сентября 2016 г.

В окончательной редакции 22 декабря 2016 г.)

C использованием программного пакета WIEN2k проведено ab initio моделирование равновесной структуры и свойств ГЦК-железа с примесью углерода. Предложена методика, позволяющая провести моделирование магниторазупорядоченного состояния системы в рамках теории функционала плотности. В рамках данной методики проведен расчет величины энергии растворения углерода, которая составила $0.25 \mathrm{eV}$. Определены также энергии взаимодействия между атомами углерода, находящимися в первой, второй и третьей координационных сферах друг друга, которые составили $E_{1}=0.06 \mathrm{eV}, E_{2}=0.1 \mathrm{eV}$ и $E_{3}=0.005 \mathrm{eV}$. Для проверки достоверности полученных значений энергий проведен расчет активности углерода методом Монте-Карло. Хорошее качественное согласие рассчитанной активности с экспериментальными данными указывает на достоверность полученных энергетических параметров.

Настоящая работа поддержана РНФ (грант № 16-19-10252).

DOI: 10.21883/FTT.2017.07.44583.342

\section{1. Введение}

Благодаря высокой прочности стали остаются основными конструкционными материалами, несмотря на развитие производства пластмасс, керамики, композитов. Основой любой стали является твердый раствор углерода в железе. Прочностные свойства стали существенно зависят от структурных особенностей этого раствора, которые в свою очередь определяются характером взаимодействия между атомами углерода в решетке железа. Железо является полиморфным металлом $[1,2]$ : при нагревании выше $911^{\circ} \mathrm{C}$ низкотемпературное $\alpha$-железо с ОЦК-решеткой превращается в $\gamma$-железо с ГЦК-решеткой.

Обычно используются стали на основе низкотемпературной ОЦК-фазы железа. Морфология ее микроструктуры, имеющая решающее значение для всех практических приложений, образуется в процессе закалки в области высоких температур, где железо существует в ГЦК-фазе. Вопрос о взаимодействии между атомами углерода в аустените играет очень важную роль для физического металловедения. Во-первых, в условиях быстрого охлаждения аустенита, когда диффузия углерода подавлена, происходит бездиффузионное мартенситное превращение [2], при котором все атомы углерода принудительно переходят в решетку мартенсита, наследуя распределение материнской фазы. Во-вторых, растворимость углерода в цементите очень низка [2], что затрудняет экспериментальное изучение взаимодействия между атомами С в ОЦК-решетке. Растворимость же углерода в ГЦК-решетке аустенита значительно выше, что позволило достаточно надежно изучить кон- центрационные зависимости ряда термодинамических характеристик.

К сожалению, энергии взаимодействия атомов растворенного вещества не могут быть непосредственно измерены в эксперименте. Поэтому они могут быть оценены с помощью термодинамических моделей $[3,4]$ на основе фазовых диаграмм или данных о параметрах ближнего порядка [5-7] либо методами ab initio компьютерного моделирования [8].

ОЦК-железо является ферромагнитным, и его моделирование не представляет проблем [9-11]. ГЦК-фаза железа стабильна в температурной области, лежащей выше точки Кюри, где наблюдается парамагнитное состояние. Реализация в современных вычислительных пакетах теории функционала плотности проводится с использованием приближения локальной спиновой плотности. В рамках такого приближения неколлинеарные магнитные конфигурации не могут быть рассмотрены, поэтому возникает важный вопрос: какая из моделей коллинеарного магнитного упорядочения лучше всего описывает физические свойства ГЦК-железа?

Основными магнитными состояниями, используемыми для моделирования парамагнитного ГЦК-железа, являются немагнитное (НM), ферромагнитное высокоспиновое (ФМВС) и ферромагнитное низкоспиновое $(\Phi \mathrm{MHC})$, антиферромагнитное однослойное (АФМ1) и антиферромагнитное двухслойное (АФМД). Эти магнитные состояния приведены на рис. 1. В НМ-состоянии железа магнитный момент равняется нулю. В ферромагнитном состоянии все спины атомов железа направлены в одну сторону. В антиферромагнитном состоянии чередуются слои: в однослойном - слой атомов железа со 


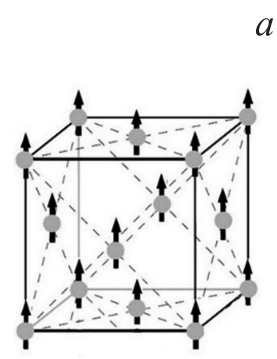

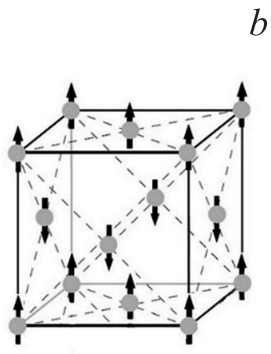

$b$

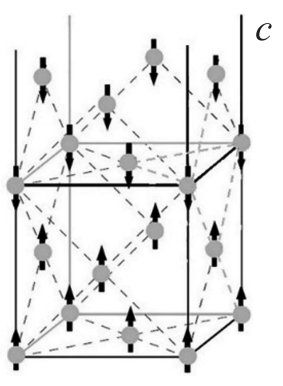

Рис. 1. Магнитные состояния в ГЦК-железе: $a-$ ферромагнитное, $b-$ антиферромагнитное однослойное, $c$ - антиферромагнитное двухслойное. Стрелками показаны направления спинов атомов железа.

спином вверх и слой со спином вниз, а в двухслойном два слоя атомов железа со спином вверх и два со спином вниз.

Растворение углерода в ГЦК-железе представляет собой эндотермическую реакцию (протекает с поглощением тепла). Энергия этого процесса была вычислена экспериментально в работах [12-16], на основе анализа которых можно сделать вывод, что ее величина лежит в интервале от 0.36 до $0.48 \mathrm{eV}$. Некоторые авторы использовали НМ-состояния для моделирования парамагнитного состояния, поскольку считали, что в парамагнитном состоянии магнитный момент равняется нулю и его можно использовать для моделирования парамагнитного состояния [17,18]. Ферромагнитное состояние разделяется на два магнитных состояния с большим и маленьким спином: ФМВС и ФМНС. Существуют модели, в которых считается, что реальное парамагнитное ГЦК-железо является их смесью либо смесью ФМВС- и АФМД-состояний [19], но эти модели не подтверждены экспериментом.

Использование АФМД-состояния для описания парамагнитного состояния ГЦК-железа поддерживают авторы работ [20,21]. Медведева и др. в [21] получили что АФМД-состояние лежит ниже по энергии, чем НМ-, ФМНС-, ФМВС- и АФМ1-состояния, и поэтому является наиболее стабильным. В работе же [20] Бухвалов и др. проводили моделирование энергии растворения углерода в АФМД-состоянии ГЦК-железа и получили достаточно близкое к экспериментальному значение $0.55 \mathrm{eV}$. Однако ряд авторов [10,22] считает, что АФМ1-состояние также вполне удовлетворительно описывает свойства парамагнитного ГЦК-железа. В работе Хэрпера и др. [22] методом FLAPW было найдено, что основное состояние ГЦК-Fе является антиферромагнитным, причем обе антиферромагнитные структуры имеют близкие значения энергии, хотя АФМДфаза имеет больший объем, значение которого лучше согласуется с данными эксперимента. Этот вывод поддерживают Хэпбурн с соавторами [10]. В своей работе они показали, что оба магнитных состояния (АФМД и АФМ1) подходят для описания парамагнитного ГЦК- железа. По данным этой работы энергия растворения углерода в АФМ1-состоянии составила $0.263 \mathrm{eV}$, а в АФМД-состоянии - $0.323 \mathrm{eV}$, что неплохо согласуется с экспериментальными значениями (в работе использована суперьячейка из 256 атомов). Следует, однако, отметить, что данный вывод базируется на расчетах, использующих метод псевдопотенциала. Основной недостаток такого моделирования состоит в том, что его результаты существенно зависят от выбора используемого псевдопотенциала, осуществляемого подгонкой к существующим экспериментальным данным. Если разброс имеющихся данных велик, то выбор потенциала становится затруднительным и предсказательная точность метода снижается. Полнопотенциальные методы расчета лишены этого недостатка, поскольку в них все базисные орбитали - как валентные, так и остовные определяются самосогласованным решением уравнений Кона-Шэма. Также необходимо учесть, что в АФМ1и АФМД-состояниях наблюдается тетрагональное искажение решетки, т.е. тетрагональная ГЦК-фаза имеет более низкую энергию, чем кубическая. В связи с этим в настоящей работе расчет энергий растворения атомов углерода в ГЦК-железе и взаимодействия между ними проведен для наиболее известных существующих упорядоченных магнитных состояний в ГЦК-железе, а также в схеме отбора магнитонеупорядоченных структур (MSM) [23]. Сравнение полученных результатов позволит определить оптимальный подход для расчета энергетических параметров углерода в парамагнитной фазе ГЦК-железа.

\section{2. Методика расчета}

2.1. Параметры моделирования. Расчеты проводились $a b$ initio полнопотенциальным методом LAPW c учетом обобщенного градиентного приближения PBE-GGA в программном пакете WIEN2k, обеспечивающем высокую точность расчета полной энергии при минимальном количестве подгоночных параметров. Это наиболее точный метод, используемый в рамках теории функционала плотности [24]. Для интегрирования в обратном пространстве и вычисления электронной плотности использовалась схема Монхорста-Пака [25] с сеткой $4 \times 4 \times 4 k$-точек в зоне Бриллюэна для оптимизации геометрических параметров и сеткой из $7 \times 7 \times 7$ $k$-точек при проведении расчетов, что обеспечивает точность расчета энергии системы в $0.01 \mathrm{eV}$. Основные параметры моделирования следующие [26]: $R_{\mathrm{MT}} K_{\max }=6$ (для систем без углерода $R_{\mathrm{MT}} K_{\max }=10$ ), радиусы muffin-tin cфep $R_{\mathrm{MT}}(\mathrm{Fe})=2.00$ a.u, $R_{\mathrm{MT}}(\mathrm{C})=1.20$ a.u.

2.2. Расче т в различных упорядоченных магнитных состояниях. Расчеты проводились в суперьячейке из 32 атомов железа. Соответствующий магнитный порядок задавался искусственно в качестве начального условия. В НМ-состоянии магнитные моменты атомов железа задавались равными нулю. При 
расчете в ферромагнитном состоянии все магнитные моменты атомов железа ориентировались вдоль одного направления. В АФМ1-состоянии искусственно чередовались слои атомов железа со спином вверх и со спином вниз. Аналогично в АФМД-состоянии чередовались два слоя атомов железа со спином вниз и два слоя атомов железа со спином вверх. Для всех типов магнитного порядка проводилась объемная минимизация параметра решетки, а затем атомно-силовая релаксация конфигурации системы, при которой исходный магнитный порядок сохранялся. Различные магнитные моменты атомов железа в ФМНС- и ФМВСсостояниях, как и ожидалось, были получены вследствие изменения объема системы. При вычислении энергии растворения использовались конфигурации после релаксации, соответствующие минимальной энергии. Полученные при этом равновесные значения параметров решетки находятся в хорошем согласии с данными работ $[9,10,20-22]$. Отметим, что магнитная структура может реализоваться как в кубических решетках (НM, ФМВС, ФМНС, АФМ1, АФМД), так и в тетрагональных решетках (АФМ1-т, АФМД-т), причем тетрагональные состояния являются более низколежащими по энергии. Поэтому в настоящей работе помимо всех наиболее известных магнитных кубических состояний для описания парамагнитного состояния ГЦК-железа использовалось также антиферромагнитное двухслойное тетрагональное состояние АФМД-т, для которого объемная оптимизация проводилась.

2.3. Методика расчета неупорядоченного парамагнитного состояния. Температурный диапазон существования ГЦК-железа лежит выше $1000 \mathrm{~K}$, но используемая в рамках программного пакета WIEN2k теория функционала плотности позволяет провести моделирование только основного состояния системы при 0 К. При объемной минимизации по энергии нами было получено, что происходит образование упорядоченных магнитных структур, которые не позволяют учесть наличие магнитного беспорядка. Кроме того, в высокотемпературной парамагнитной фазе возникают различные локальные магнитные структуры (локальные флуктуации магнитного порядка). Если атом углерода попадает в области с разным магнитным порядком, он может обладать различной энергией растворения, а средняя энергия по сплаву будет представлять собой усредненное значение по таким локальным параметрам (влияние локальных флуктуаций магнитного порядка). Для учета указанных эффектов в настоящей работе предложена модель парамагнитного состояния, основанная на схеме отбора MSM-структур. Близкая схема использована ранее в работе Пономарёвой и др. [8].

Для учета эффектов температурного расширения при моделировании проводилась атомно-силовая релаксация системы, без объемной минимизации. При этом использовались экспериментальные значения параметров решетки, взятые из экспериментальной работы Онинка [27] для $T=1400 \mathrm{~K}: a=3.667 \AA$ для чистого ГЦК-Fе, $a=3.687 \AA$ для системы из 32 атомов железа и растворенного атома углерода и $a=3.707 \AA$ для системы из 32 атомов железа и двух растворенных атомов углерода.

Различные неэквивалентные магнитные конфигурации были получены с помощью программы BINAR [28]. B основе алгоритма программы BINAR лежит анализ степени неупорядоченности множества случайных конфигураций, полученных „вбрасыванием“ атомов железа со спином вверх в подрешетку атомов железа со спином вниз с использованием генератора случайных чисел (в нашей суперьячейке из 32 атомов 16 атомов со спином вверх и 16 со спином вниз). После этого производится отбраковка конфигураций, не соответствующих критерию неупорядоченности. В качестве такого критерия используется величина суммы квадратов отклонений числа разнородных пар атомов (первый сорт атомов атомы железа со спином вверх, второй сорт - атомы железа со спином вниз) во второй координационной сфере для рассматриваемой конфигурации от статистических средних значений для полностью неупорядоченной бинарной смеси (критерий согласия Пирсона $\chi^{2}$ ). В результате определялись магнитные конфигурации, в наибольшей степени соответствующие неупорядоченной ориентации атомных магнитных моментов по узлам суперъячейки и обеспечивающие равенство нулю полного магнитного момента ячейки.

B настоящей работе, как и в предыдущей [29], с помощью программы BINAR [28] было получено 16 различных неэквивалентных магнитных конфигураций. По известной разности полной энергии $n$-й конфигурации системы и энергии системы в наинизшем основном состоянии была вычислена каноническая статистическая сумма $S$

$$
S=\sum_{n=1}^{5} \exp \left(-\left(E_{n}-E_{0}\right) / k T\right),
$$

где $E_{0}$ - энергия основного состояния, $E_{n}-$ энергия системы в $n$-м энергетическом состоянии, $k$ - коэффициент Больцмана, $T=1400 \mathrm{~K}$ (средняя температура существования ГЦК-фазы железа).

Из отношения вклада в статистическую сумму от данной конфигурации $S_{n}$ ко всей статистической сумме была вычислена вероятность существования магнитного состояния

$$
P_{n}=\frac{S_{n}}{S}
$$

Это позволяет вычислить усредненное по магнитным конфигурациям значение полной энергии суперъячейки ГЦК-матрицы железа, содержащей один или два внедренных атома углерода, по формуле

$$
\langle E\rangle=\sum_{n} E_{n} P_{n} .
$$

Для расчетов выбиралось пять лежащих ниже остальных по энергии магнитных конфигураций (рис. 2). Магнитные конфигурации низкоэнергетических состояний 


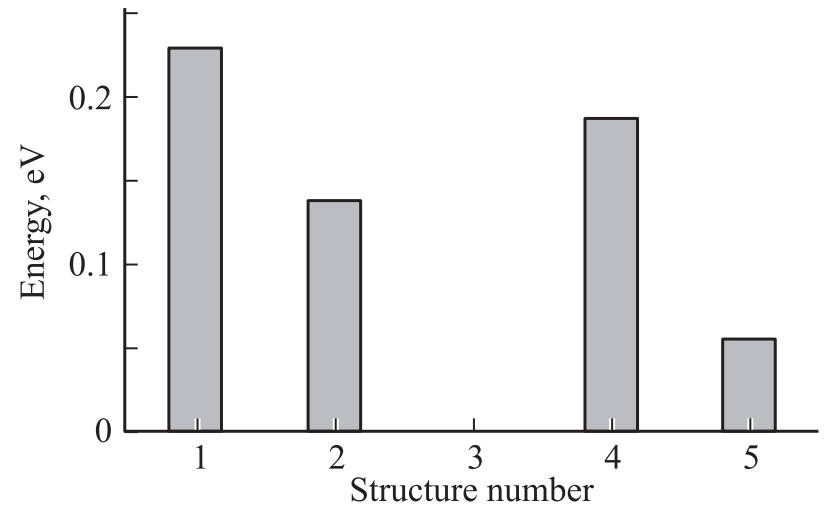

Рис. 2. Зависимость энергии системы от номера конфигурации. Энергии систем указаны относительно энергии самой низкой конфигурации.

напоминают суперъячейку АФМД-структуры, в каждой плоскости которой в среднем у двух атомов из восьми магнитные моменты перевернуты относительно ориентации остальных атомов. В высокоэнергетических же состояниях магнитные конфигурации полностью разупорядочены. Вероятность существования остальных конфигураций была меньше $3 \%$, и их учет не повлиял на конечные результаты, поэтому с целью оптимизации расчетов в дальнейшем они не учитывались.

Для тестирования методики был проведен расчет энергии растворения углерода в парамагнитном состоянии ГЦК-железа (табл. 1), величина которой оказалась равной $0.25 \pm 0.03 \mathrm{eV}$.

Таблица 1. Энергия растворения углерода в ГЦК-железе в различных магнитных конфигурациях

\begin{tabular}{c|c|c}
\hline $\begin{array}{c}\text { Номер } \\
\text { конфигурации }\end{array}$ & Вероятность, \% & $\begin{array}{c}\text { Энергия } \\
\text { растворения, } \mathrm{eV}\end{array}$ \\
\hline 1 & 6 & -0.08 \\
2 & 14 & 0.24 \\
3 & 44 & 0.3 \\
4 & 9 & 0.19 \\
5 & 27 & 0.29 \\
$\langle E\rangle$ & 100 & $0.25 \pm 0.03$
\end{tabular}

2.4. Методика расчета активности углерода в ГЦК-железе. Для моделирования активности углерода в ГЦК-железе мы использовали хорошо известный метод, предложенный в [30] и использованный для расчетов активности углерода в железе в работах $[5,6]$. Расчет активности углерода в ГЦК-железе проводился по формуле

$$
a_{\mathrm{C}}=a_{\mathrm{conf}} \exp (\Delta G / k T),
$$

где $a_{\mathrm{C}}-$ активность углерода в ГЦК-железе, $a_{\text {conf }}-$ конфигурационная часть активности, а $\exp (\Delta G / k T)-$ ее неконфигурационная часть. Для расчета последней использовались два набора данных из работ $[5,6]$. При этом в зависимости от используемого набора возникали различия в величине неконфигурационного множителя порядка 10-20\%. Нами был взят набор из работы [5], потому что в ней при вычислении экспоненты напрямую использовались экспериментальные данные об активности [31].

Алгоритм вычисления конфигурационной части $a_{\text {conf }}$ детально описан в работах $[5,6,30]$. Идея метода заключается в следующем. Для каждого члена статистического ансамбля при моделировании генерируется лишь часть суммы (виртуальный ввод одного атома углерода при фиксированной позиции других). Эта сумма усредняется при использовании обычной процедуры Монте-Карло. Выражение для $a_{\text {conf }}$ имеет вид

$$
a_{\mathrm{conf}}=M\left(N_{\mathrm{C}}+1\right) /\left\{\sum_{i=1}^{M}\left[\sum_{j}^{\mu} \exp \left(-\Delta E_{j} / k T\right)\right]\right\},
$$

где выполняется суммирование по $j$ по всем пустым октаэдрическим междоузлиям и по числу шагов $i(i=1,2, \ldots, M)$, используемых в расчете методом Монте-Карло; $\Delta E_{j}$ - это изменение энергии системы, когда вводится один виртуальный атом углерода; $\mu=N_{\mathrm{Fe}}-N_{C}-$ число междоузлий в кристалле.

\section{3. Результаты}

Для определения энергии растворения углерода относительно фазы графита использовалась формула

$$
\Delta H=E_{\mathrm{Fe}_{32} \mathrm{C}}-E_{\mathrm{Fe}_{32}}-E_{\mathrm{C}},
$$

где $E_{\mathrm{Fe}_{32} \mathrm{C}}-$ энергии суперьячейки после релаксации, состоящей из 32 атомов железа и одного атома углерода, находящегося в октапоре, $E_{\mathrm{Fe}_{32}}-$ энергия суперъячейки, состоящей из 32 атомов железа, а $E_{\mathrm{C}}-$ энергия одного атома углерода в решетке графита. Параметры структуры графита были взяты из работы [9] $(a=2.462 \AA$, $\left.c=6.656 \AA, \alpha=90^{\circ}, \beta=90^{\circ}, \gamma=120^{\circ}\right)$. Значения энергий растворения углерода в различных магнитных фазах ГЦК-железа представлены в табл. 2.

Таблица 2. Энергия растворения углерода в ГЦК-железе в различных магнитных состояниях (в еV)

\begin{tabular}{c|c|c}
\hline Состояние & Данные других авторов & Наст. раб. \\
\hline НМ & $0.145[17]$ & 0.18 \\
ФМНС & - & -1.25 \\
ФМВС & $-0.17[9]$ & -0.11 \\
АФМ1 & - & -0.33 \\
АФМ1-т & $0.263[10]$ & - \\
АФМД & - & 0.34 \\
АФМД-т & $0.323[10], 0.55[20]$ & 0.47 \\
Парамагнитное & $0.2[8]$ & 0.25 \\
Эксперимент & $0.36-0.48[12-16]$ & -
\end{tabular}




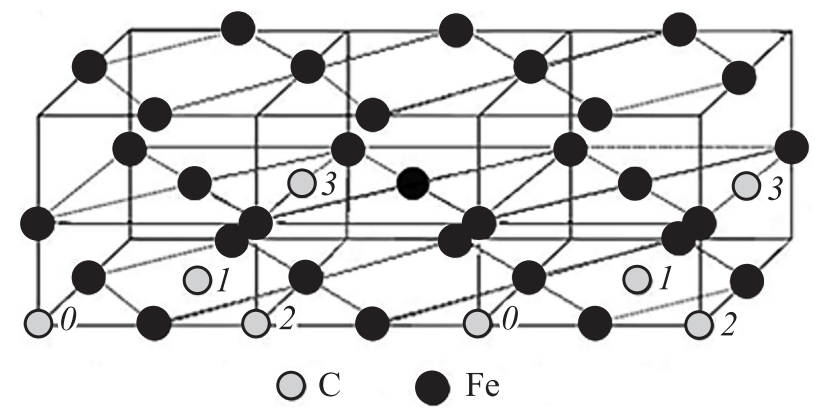

Рис. 3. Расположение атомов углерода в суперьячейке из 32 атомов железа с учетом периодических граничных условий. Новая суперьячейка начинается от второго атома номер 0 .

На основе анализа этой таблицы можно сделать вывод, что используемый набор параметров моделирования обеспечивает неплохое совпадение результатов с данными других авторов при описании основных магнитных фаз (немагнитной, ферромагнитной и антиферромагнитной) для ГЦК-решетки железа. Поэтому неудивительно, что наши результаты хорошо воспроизводят литературные данные для магнитных структур АФМД-т и MSM, используемых для описания парамагнитной фазы железа. Видно, что наилучшее согласие с экспериментом достигается при использовании для моделирования коллинеарной магнитной структуры АФМД-т и неупорядоченной структуры, полученной методом MSM. Поэтому расчеты энергии взаимодействия между парой атомов углерода, растворенных в парамагнитной фазе железа, были проведены именно для этих моделей, что делает возможным их дальнейшее сопоставление.

Для нахождения энергии взаимодействия между атомами углерода в ГЦК-железе использовалась формула

$$
\Delta E=E_{\mathrm{Fe}_{32} \mathrm{C}_{2}}-2 E_{\mathrm{Fe}_{32} \mathrm{C}}+E_{\mathrm{Fe}_{32}},
$$

где $E_{\mathrm{Fe}_{32} \mathrm{C}_{2}}-$ энергия суперьячейки после релаксации, состоящей из 32 атомов железа и двух атомов углерода, находящихся в октапорах. Первый атом помещался в октапору, находящуюся в начале координат (позиция 0 на рис. 3), а второй атом последовательно помещался в первую, вторую и третью координационные сферы относительно первого (позиции 1,2,3 на рис. 3). Отметим, что найденную в нашем случае энергию взаимодействия во второй и третьей координационных сферах необходимо разделить пополам. Это связано с тем, что при расчете используются периодические граничные условия, а эти позиции (рис. 3) находятся на равном удалении от атома углерода в начале координат и его периодического повторения, возникающего при периодической трансляции ячейки. В силу этого рассчитанная энергия оказывается равна удвоенной энергии взаимодействия между изолированной парой атомов углерода.
Энергии взаимодействия между атомами углерода для первых двух координационных сфер в ГЦК-железе можно оценить косвенным путем на основе экспериментальных исследований [3-7]. Многочисленные термодинамические расчеты $[3,4]$, основанные на различных модельных представлениях о взаимодействии между атомами углерода, приводят к сильно различающимся результатам. Однако все они указывают на достаточно сильный отталкивательный характер этого взаимодействия в ближайших координационных сферах. Наиболее точные значения энергий взаимодействия между атомами углерода в ГЦК-железе получены на основе моделирования методом Монте-Карло мессбауэровских спектров ГЦК-сплавов $\mathrm{Fe}-\mathrm{C}$ [5-7]. Все указанные литературные данные о значениях энергии взаимодействия между атомами углерода, а также результаты, полученные в настоящей работе, представлены в табл. 3.

Из табл. 3 видно, что в соответствии с нашими расчетами атомы углерода отталкиваются в первой и второй координационных сферах, причем во второй координационной сфере сильнее, чем в первой, что согласуется с данными [3-7]. В работе же [8], в которой также использовался MSM-метод [23], наблюдается обратное. Это отличие, вероятно, связано с тем, что нами использовался полнопотенциальный пакет WIEN2k, тогда как в [8] применялся псевдопотенциальный пакет VASP. Чтобы окончательно убедиться в адекватности полученных нами значений энергий взаимодействия, был проведен расчет активности углерода в ГЦК-железе. Применялся метод, описанный в подразделе 2.4, причем для расчета использовались как набор энергий взаимодействия углерод-углерод, полученный нами, так и данные [8]. Результаты моделирования приведены на рис. 4, из которого видно, что активность, полученная по нашим данным, значительно лучше совпадает с активностью, установленной экспериментально

Таблица 3. Энергии взаимодействия между атомами углерода в ГЦК-железе (в еV)

\begin{tabular}{|c|c|c|c|}
\hline \multicolumn{3}{|c|}{ Номер координационной сферы } & \multirow{2}{*}{$\begin{array}{l}\text { Лит. } \\
\text { ссылка }\end{array}$} \\
\hline 1 & 2 & 3 & \\
\hline-0.01 & -0.015 & 0.035 & $\begin{array}{l}\text { Наст. раб. } \\
\text { (АФМД-т) }\end{array}$ \\
\hline 0.06 & 0.1 & 0.005 & $\begin{array}{l}\text { Наст. раб. } \\
\text { (MSM) }\end{array}$ \\
\hline 0.6 & - & - & [3] \\
\hline 0.08 & - & - & [4] \\
\hline 0.115 & 0.169 & - & {$[5]$} \\
\hline 0.036 & 0.075 & - & [6] \\
\hline 0.1 & - & - & [7](модель 1) \\
\hline $0-0.075$ & $>0.045$ & - & [7] (модель 2) \\
\hline$-0.015-0.045$ & $>0.08$ & - & [7] (модель 3) \\
\hline 0.18 & 0.17 & -0.004 & [8] \\
\hline
\end{tabular}




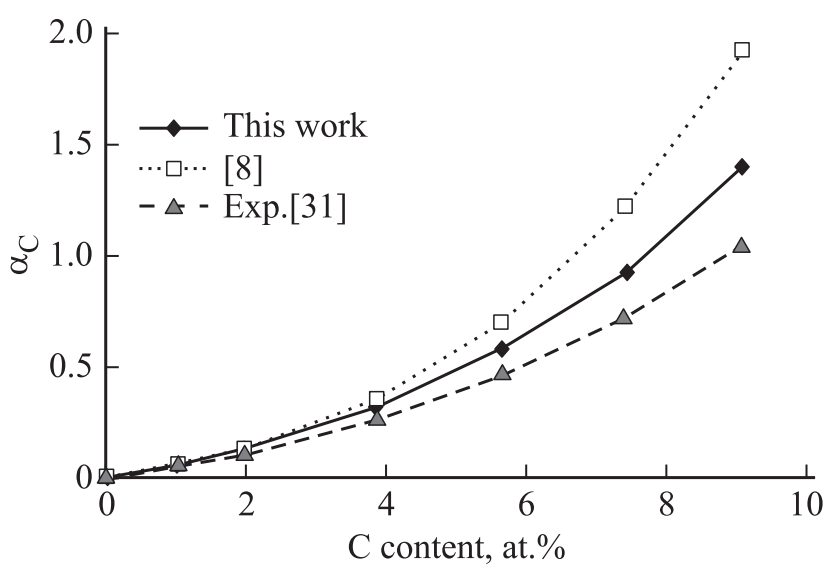

Рис. 4. Сравнение кривых активности, полученных при компьютерном моделировании методами Монте-Карло, с экспериментальными (при $T=1423 \mathrm{~K}$ ).

в [31], чем при использовании данных [8]. Подобные расчеты проводились для температур $T=1173$, $1273,1373,1423,1573 \mathrm{~K}$, и для всего диапазона лучшее согласие наблюдалось при использовании наших данных.

\section{4. Заключение}

В работе методом $a b$ initio моделирования получены значения энергии растворения атома углерода и энергии взаимодействия растворенных в парамагнитном ГЦК-железе пар атомов углерода, расположенных в первой, второй и третьей координационных сферах относительно друг друга. Показано, что АФМД-состояние недостаточно точно описывает парамагнитное ГЦК-железо. Наиболее сильное отталкивание наблюдается между атомами углерода, располагающимися во второй координационной сфере. Полученные нами значения энергии взаимодействия между атомами углерода существенно отличаются от значений, используемых в большинстве термодинамических моделей, однако достаточно близки к результатам $a b$ initio моделирования в работе Пономарёвой и др. [8]. Для проверки достоверности полученных значений энергий проведен расчет активности углерода методом Монте-Карло. Хорошее качественное согласие рассчитанной активности с экспериментальными данными свидетельствует о достоверности полученных энергетических параметров.

Таким образом, использованная нами методика расчета парамагнитного состояния позволяет получить адекватные энергию растворения атомов углерода, энергетические характеристики взаимодействия между атомами углерода в ГЦК-железе, что подтверждается качественным согласием расчетов активности углерода в аустените с данными эксперимента.

\section{Список литературы}

[1] Я.С. Уманский, Ю.А. Скаков, А.Н. Иванов, Л.Н. Расторгуев. Кристаллография, рентгенография и электронная микроскопия. Металлургия, М. (1982). 632 с.

[2] Г.В. Курдюмов, Л.М. Утевский, Р.И. Энтин. Превращения в железе и стали. Наука, М. (1977). 236 с.

[3] Б.М. Могутнов, Н.А. Томилин, Л.А. Шварцман. Термодинамика сплавов железа. Металлургия, М. (1984). 208 с.

[4] R.B. Mclellan. Acta Met. 35, 2151 (1987).

[5] M.S. Blanter. J. Alloys Compd. 291, 167 (1999).

[6] A.L. Sozinov, A.G. Balanyuk, V.G. Gavriljuk. Acta Mater. 45, 225 (1997).

[7] A.G. Balanyuk, V.N. Bugaev, V.M. Nadutov, A.L. Sozinov. Phys. Status Solidi B 207, 3 (1998).

[8] А.В. Пономарёва, Ю.Н. Горностырёв, И.А. Абрикосов. ЖЭТФ 147, 4, 827 (2015).

[9] D.E. Jiang, E.A. Carter. Phys. Rev. B 67, 214103 (2003).

[10] D.J. Hepburn, D. Ferguson, S. Gardner, G.J. Ackland. Phys. Rev. B 88, 024115 (2013).

[11] C. Domain, C.S. Becquart, J. Foct. Phys. Rev. B 69, 144112 (2004).

[12] P. Gustafson. Scand. J. Met. 14, 259 (1985).

[13] Б.М. Могутнов, И.А. Томилин, Л.А. Шварцман. Термодинамика сплавов железа. Металлургия, М. (1984). 206 с.

[14] A. Lobo, G.H. Geiger. Met. Trans. A 7, 1359 (1976).

[15] G.J. Shiflet, J.R. Bradley, H.I. Aaronson. Met. Trans. A 15, 1287 (1984).

[16] J. Chipman. Met. Trans. 3, 55 (1972).

[17] J.A. Slane, C. Wolverton, R. Gibala. Met. Mater. Trans. A 35, 2239 (2004).

[18] N.I. Medvedeva, M.S. Park, D.C.V. Aken, J.E. Medvedeva. J. Alloys Compd. 582, 475 (2014).

[19] M. Acet, H. Zahres, E.F. Wassermann, W. Pepperhoff. Phys. Rev. B 49, 6012 (1994).

[20] D.W. Boukhvalov, Y.N. Gornostyrev, M.I. Katsnelson, A.I. Lichtenstein. Phys. Rev. Lett. 99, 247205 (2007).

[21] N.I. Medvedeva, D.V. Aken, J.E. Medvedeva. J. Phys. Condens. Matter. 22, 316002 (2010).

[22] H.C. Herper, E. Hoffmann, P. Entel. Phys. Rev. B 60, 3839 (1999).

[23] B. Alling, T. Marten, I.A. Abrikosov. Phys. Rev. B 82, 184430 (2010).

[24] S. Cottenier. Density functional theory and the family of (L)APW-methods: a step-by-step introduction. (2004). $65 \mathrm{p}$.

[25] H.J. Monkhorst, J.D. Pack. Phys. Rev. B 13, 5188 (1976).

[26] Я.М. Ридный, А.А. Мирзоев, Д.А. Мирзаев. Вестн. ЮУрГУ. Cер. Математика. Механика. Физика 5, 2, 108 (2013).

[27] M. Onink, C.M. Brakman, F.D. Tichelaar, E.J. Mittemeijer, S. van der Zwaag, J.H. Root, N.B. Konyer. Scripta Met. Mater. 29, 1011 (1993).

[28] Р.З. Деянов, Н.Н. Еремин, В.С. Урусов. ODSS (OrderedDisordered-Solid-Solution). Ver.1.-binar Программа расчета неупорядоченных сверхъячеек для моделирования твердых растворов замещения. М. (2006-2007). 48 с.

[29] Я.М. Ридный, А.А. Мирзоев, Д.А. Мирзаев. Вестн. ЮУрГУ. Сер. Математика. Механика. Физика 6, 3, 86 (2014).

[30] E. Murch, R.J. Thorn. Acta Met. 27, 201 (1979).

[31] S. Ban-ya, J.F. Elliott, J. Chipman. Met. Trans. 1, 1313 (1970). 